\title{
Effects of graft preconditioning on $\gamma$-irradiated deep frozen tendon allografts used in anterior cruciate ligament reconstruction
}

\author{
XIAOZUO ZHENG ${ }^{1,2^{*}}$, WEI XU ${ }^{1,3^{*}}$, JUYUAN GU ${ }^{1,2}$, YANG HU ${ }^{4}$, \\ MEIJUAN CUI ${ }^{5}$, YU-E FENG ${ }^{1}$ and SHIJUN GAO ${ }^{1,2}$
}

\author{
${ }^{1}$ Department of Orthopedics; ${ }^{2}$ Orthopaedic Biomechanics Laboratory of Hebei Province, \\ Third Hospital of Hebei Medical University, Shijiazhuang, Hebei 050051; ${ }^{3}$ Emergency Department, \\ Second Hospital of Hebei Medical University, Shijiazhuang, Hebei 050000; ${ }^{4}$ The Second Department of \\ Intensive Care Unit, Children's Hospital of Hebei Province, Shijiazhuang, Hebei 050000; ${ }^{5}$ Department of \\ Medical Records, Third Hospital of Hebei Medical University, Shijiazhuang, Hebei 050051, P.R. China
}

Received December 5, 2017; Accepted May 25, 2018

DOI: $10.3892 /$ etm.2018.6338

\begin{abstract}
Preconditioning of the grafts prior to implantation into the knee is considered to reduce the loss of tension caused by graft viscoelasticity after anterior cruciate ligament reconstruction. The present study analyzed the impacts of different preconditioning forces on the biomechanical properties of the $\gamma$-irradiated deep frozen tendon allografts. A total of 36 tendon grafts were randomly divided into three groups and were preconditioned at $80 \mathrm{~N}$ (group 1), $160 \mathrm{~N}$ (group 2) and $320 \mathrm{~N}$ (group 3) for $10 \mathrm{~min}$. Subsequently, the grafts were gradually completely relaxed for $1 \mathrm{~min}$ and subsequently received 25 cyclic loads of $0-80 \mathrm{~N}$. Afterwards, the grafts were loaded to $80 \mathrm{~N}$, which was maintained for $30 \mathrm{~min}$. Finally, load was gradually increased until ultimate failure at maximum load (UFML) was obtained. There were significant differences in the stiffness and UFML values between the 3 groups (all $\mathrm{P}<0.05$ ). The graft stiffness in group 3 significantly increased compared with the other 2 groups, and the stiffness of group 2 grafts increased compared with group 1 . The UFML in group 3 was significantly lower compared with groups 1 and 2 , while there was no significant difference between groups 1 and 2. In the present study, the results suggested that increasing the initial
\end{abstract}

Correspondence to: Dr Xiaozuo Zheng, Department of Orthopedics, Third Hospital of Hebei Medical University, 139 Ziqiang Road, Shijiazhuang, Hebei 050051, P.R. China

E-mail: xiaozuo_zheng@163.com

Dr Shijun Gao, Orthopedic Biomechanics Laboratory of Hebei Province, Third Hospital of Hebei Medical University, 139 Ziqiang Road, Shijiazhuang, Hebei 050051, P.R. China

E-mail: 13595933@qq.com

*Contributed equally

Key words: allograft, graft preconditioning, anterior cruciate ligament reconstruction, mechanics, tendons tension could effectively reduce the loss of stiffness due to viscoelasticity for the $\gamma$-irradiated deep frozen allogeneic tendon grafts. However, overloaded initial tension decreased the tensile strength.

\section{Introduction}

Arthroscopic anterior cruciate ligament (ACL) reconstruction has become a primary method for the treatment of ACL rupture (1). There are numerous factors that may influence the success of ACL reconstruction, including graft selection, the choice of surgical technique (2), bone tunnel location, graft fixation method and rehabilitation protocol. Biomechanical properties of soft tissue grafts, represented by the levels of viscoelasticity, serve a role in determining the long-term results (1). Tendon grafts used in ACL reconstruction relax and elongate due to viscoelasticity, which may result in a postoperative decrease in the graft tension (3). Preconditioning is therefore recommended prior to implantation, as a countermeasure to this undesired effect, to minimize the viscoelasticity-induced graft elongation and lower the risk of knee laxity in the postoperative period (4-6).

Although the need for preconditioning of soft tissue grafts has been well established, the optimal forces have not been determined. Some investigators have suggested that $80 \mathrm{~N}$ or even lower level preconditioning was enough to produce a rigid implant and to limit postoperative knee laxity effectively (3,7-9). Others argued that increasing the tension applied to the tendon grafts could decrease the loss of tension and stiffness, and that the elongation could be eliminated during the preconditioning processes $(3,5,10)$. However, excessive pretension forces may have a negative effect on the ultrastructure of collagen and ligamentization of the grafts. A previous study demonstrated that a $500 \mathrm{~N}$ pretension force may result in loss of cohesion, integrity and parallelism of the collagen fibrils (11). Furthermore, excessive graft tension caused by the preconditioning process may lead to additional intra-articular injuries, including cartilage degeneration (12) and impairment of graft revascularization (13). Therefore, it is important to identify the suitable preconditioning forces for different types of grafts. 
The use of a tendon allograft, as a substitute for an autograft, prevents donor-site complications and has been commonly used in ACL reconstruction (14,15). The majority of the tendon allografts receive $\gamma$-ray irradiation to kill bacteria, viruses and other microorganisms, and to reduce the risk of disease transmission (16). However, the structures of irradiated tendons may change, resulting in significant alternations in biomechanical properties $(17,18)$. As a soft tissue graft, the $\gamma$-irradiated tendon is characterized by viscoelasticity. Therefore, preconditioning is also required to maintain the biomechanical strength following implantation into the knee. The present study was designed to analyze the impact of different preconditioning forces on the stiffness and ultimate failure at maximum load (UFML), and to improve the biomechanical properties of the $\gamma$-irradiated deep frozen tendon allografts used in ACL reconstruction.

\section{Materials and methods}

Graft preparation. All irradiated human hamstring tendon allografts were supplied by a certified tissue bank, with policies for serological and microbiological testing in accordance with guidelines set by the American Association of Tissue Banks (Shanxi OsteoRad Biomateral Co., Ltd., Taiyuan, China). All grafts were stored frozen at $-20^{\circ} \mathrm{C}$ for $\geq 6$ months and were radiated with $25 \mathrm{kGy}$ cobalt- 60 at room temperature for $12 \mathrm{~h}$. The $\gamma$-irradiated deep frozen tendon allografts were thawed to room temperature in $0.9 \%$ saline solution for $15 \mathrm{~min}$. Afterwards, every two of 72 frozen tendons were matched into pairs to obtain 36 tendon grafts. The tendon constructs were subsequently fixed on an ACL graft preparation table and $20 \mathrm{~mm}$ of each free end was whipstitched with Ethibond sutures (Ethibond Excel 1; Ethicon, Inc., Cincinnati, OH, USA). Subsequently, the paired tendons were folded to form 4-strand grafts with a diameter of $7 \pm 0.5 \mathrm{~mm}$. The 36 paired grafts were randomized into 3 preconditioning groups. The only difference between the 3 testing groups was the pretension force, $80 \mathrm{~N}$ (group 1), $160 \mathrm{~N}$ (group 2) and $320 \mathrm{~N}$ (group 3), representing different levels of load applied to the grafts.

Biomechanical testing. Each graft was fixed with two clamps to a biomechanical machine (Electroforce 3520; TA Instruments, New Castle, DE, USA) for testing, with $\sim 35 \mathrm{~mm}$ of distance between the two clamps (Fig. 1). An initial load of $80 \pm 0.28 \mathrm{~N}, 160 \pm 0.17 \mathrm{~N}$ and $320 \pm 0.33 \mathrm{~N}$ was applied to groups 1, 2 and 3, respectively, and maintained for $10 \mathrm{~min}$ to induce pretension of the graft on the graft board. Grafts were gradually relaxed until the load was reduced to $0 \mathrm{~N}$ and maintained for $1 \mathrm{~min}$ to mirror the process of the grafts being implanted into the knee joint. The grafts subsequently received 25 cyclic loads of 0-80 N, modeling the passive flexion-extension motion within the knee after femoral fixation. Following preconditioning, the grafts were gradually loaded to $80 \mathrm{~N}$ and the two clamps were held in place for $30 \mathrm{~min}$ to simulate the application of the initially set tension of fixing the femoral and tibial ends. Finally, the load was gradually increased until the UFML was obtained and the load vs. displacement curve was recorded (Wintest 4.1; TA Instruments). The loading profile used for each graft is illustrated in Fig. 2. The stiffness was

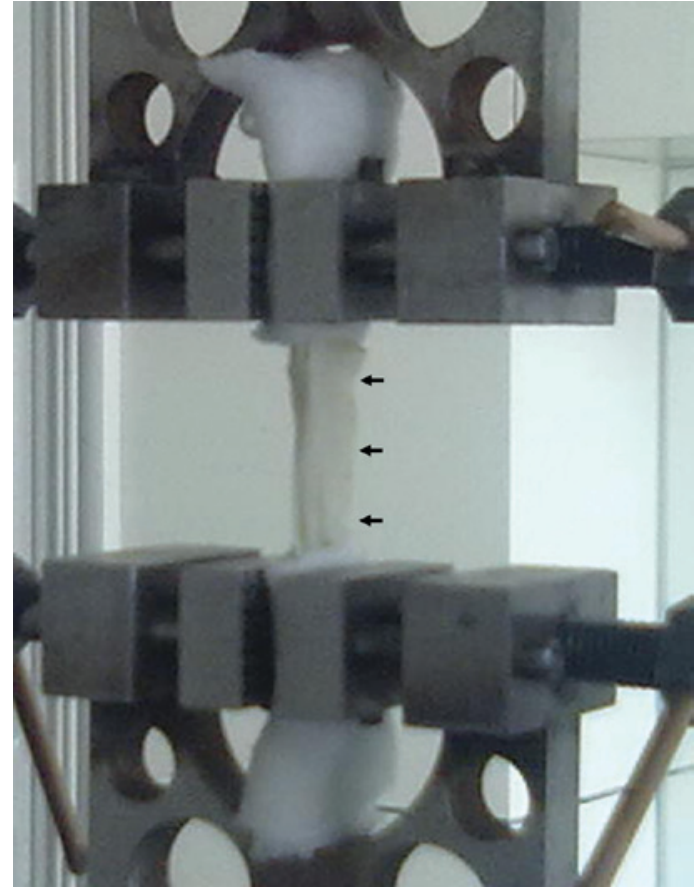

Figure 1. $\gamma$-irradiated deep frozen tendon grafts. Arrows indicating tested graft. Grafts were clamped to the actuator after being whipstitched.

calculated as the slope of the best fit through the load vs. displacement data.

Statistical analysis. All statistical analyses were performed using SPSS software version 21.0 (IBM Corp., Armonk, NY, USA). Data are presented as the mean \pm standard deviation. Differences between groups were analyzed using one-way analysis of variance and subsequently the statistical analysis was performed using the Least Significant Difference test between the different groups. $\mathrm{P}<0.05$ was considered to indicate a statistically significant difference.

\section{Results}

Stiffness. Stiffness levels of the grafts treated with different preconditioning forces are presented in Fig. 3. Statistically significant differences between the three groups were observed as the preconditioning force increased $(\mathrm{F}=25.320 ; \mathrm{P}<0.05)$. Specifically, the stiffness of grafts in group 3 increased compared with groups 1 and $2(\mathrm{P}<0.05)$ and the stiffness of grafts in group 2 increased compared with group $1(\mathrm{P}<0.05)$.

$U F M L$. UFML values of the grafts treated with different preconditioning forces are presented in Fig. 4. Statistically significant differences were observed between the three groups $(\mathrm{F}=16.662 ; \mathrm{P}<0.05)$. Specifically, the UFML value of grafts in group 3 was significantly lower compared with groups 1 and 2 (both $\mathrm{P}<0.05$ ). No significant difference was found between groups 1 and $2(\mathrm{P}=0.481)$.

\section{Discussion}

Graft selection is one of the most important factors that influence the success of ACL reconstruction (18). Autotransplantation 


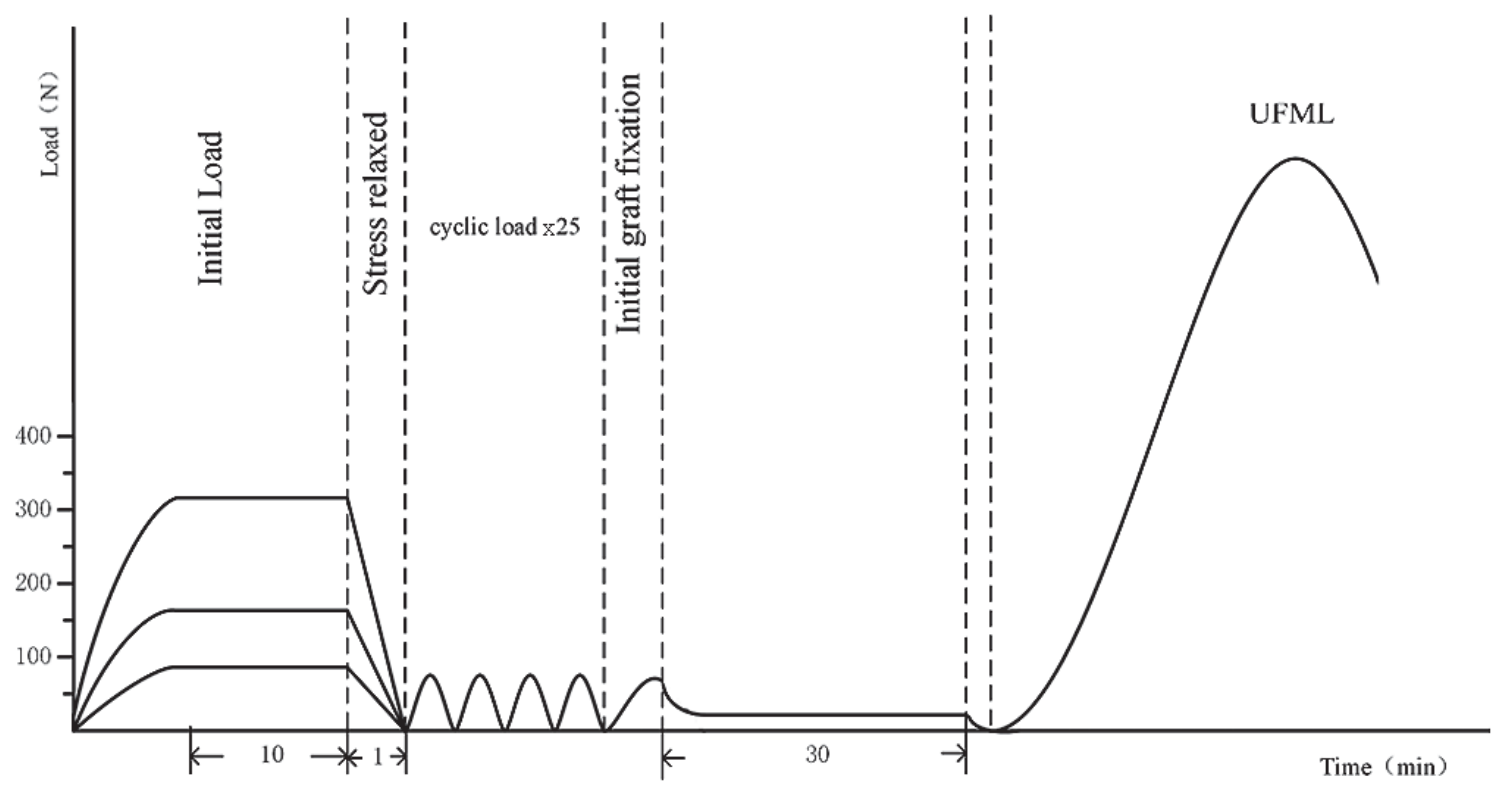

Figure 2. Schematic diagram of the loading profile for each graft tested. UFML, ultimate failure at maximum load.

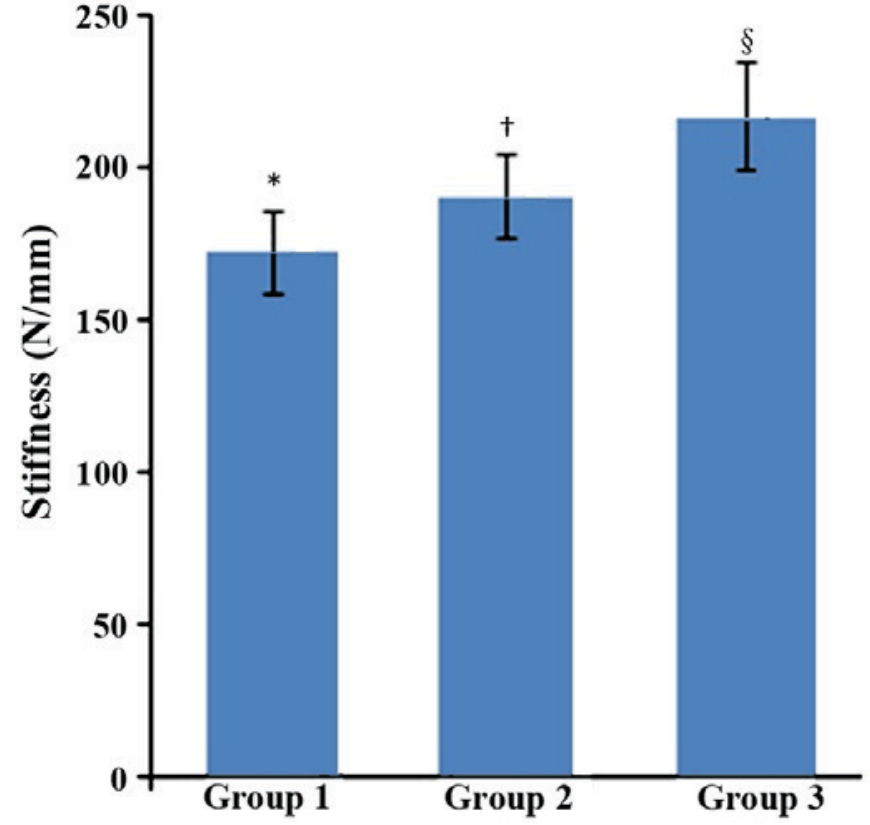

Figure 3. Stiffness of the grafts following different preconditioning protocols. ${ }^{*} \mathrm{P}<0.05$ vs. group 2 ; ${ }^{\top} \mathrm{P}<0.05$ vs. group 3 ; ${ }^{\circledR} \mathrm{P}<0.05$ vs. group 1 .

is routinely used and limited by graft resources, potential functional disability and donor site morbidity $(19,20)$. The use of allografts is a reasonable alternative to autografts and has become increasingly popular in ligament construction due to an increased availability of grafts, reduced operating time and elimination of donor-site morbidity $(18,21)$. However, there remain certain potential issues associated with allotransplantation including immune response, disease transmission and delayed ligamentization $(16,18)$. The $\gamma$-irradiation exhibits bactericidal and virucidal properties and is commonly used for the sterilization of allografts. This type of irradiation also induces adverse effects on biomechanical properties of the allografts. Previous studies have demonstrated

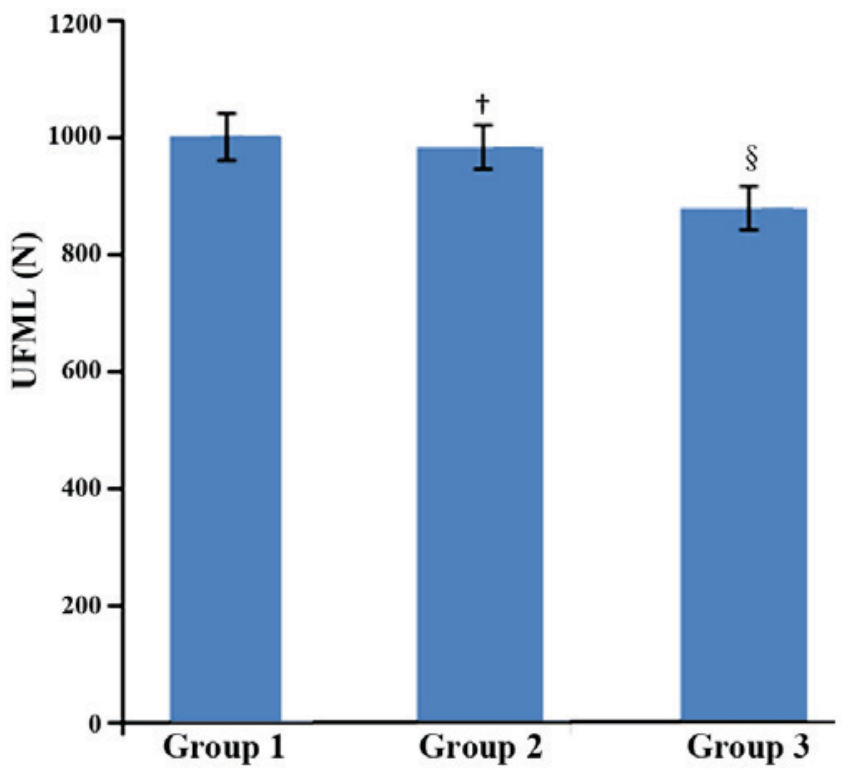

Figure 4. UFML of the grafts following different preconditioning protocols. ${ }^{\dagger} \mathrm{P}<0.05$ vs. group $3 ;{ }^{\circledR} \mathrm{P}<0.05$ vs. group 1 . UFML, ultimate failure at maximum load.

a dose-dependent association between $\gamma$-irradiation and the strength of tendon allografts $(22,23)$. Even a low-dose $(<25 \mathrm{kGy})$ of irradiation induces negative effects on the biomechanical properties of the grafts $(24,25)$. The viscoelastic character of the tendon graft inevitably changes following $\gamma$-irradiation (25). Therefore, it is important to improve the performance of the allografts used in ACL reconstruction in order to maintain the postoperative stability of the knee. The present study investigated the most commonly used frozen tendon grafts with $25 \mathrm{kGy} \gamma$-irradiation. The aim of the present study was to investigate the effects of different preconditioning load magnitudes on the stiffness and tension of $\gamma$-irradiated tendon allografts, and provide biomechanical evidence for the clinical use. 
Preconditioning is considered an effective method for eliminating graft viscoelasticity (7). As the pretension level increased during preconditioning, the stiffness of the grafts also increased. Graft tension and knee stability may benefit from the construct with a high level of stiffness (3). However, a previous study also reported that high pretension may also lead to decreased motion, delayed vascularization and graft failure (13). Furthermore, Suggs et al (26) reported that a graft with excessive stiffness may result in an overconstrained knee and suggested that intact knee kinematics could be best restored using a graft with similar stiffness to ACL (26). The present study demonstrated that an increased pretension force applied to $\gamma$-irradiated deep frozen tendon grafts during preconditioning significantly increased the stiffness of the constructs. In addition, the preconditioning protocols with load of 160 or $320 \mathrm{~N}$ seems to be more advantageous compared with that of $80 \mathrm{~N}$ for the tendon stiffness. The tendon graft stiffness preconditioned by these two protocols was closer to the native ACL (about $182-242 \mathrm{~N} / \mathrm{mm})$ that was reported in previous studies $(27,28)$.

All soft tissue implants, including ACL, undergo a similar incorporation process, including graft necrosis, cellular repopulation, revascularization and collagen remodeling (29). A previous study indicated that allografts were not as rapidly remodeled and incorporated into the host tissues as autografts (30). The tendon allograft temporarily acted as ACL prior to the completion of ligamentization. The strength of the graft decreased when implanted and then slowly recovered (31). Therefore, the tensile strength of the graft serves a role in the determination of the success of an ACL reconstruction and grafts with an increased strength are preferred (10). Although preconditioning can increase the stiffness of grafts to a certain extent, it may also affect the tensile strength. Figueroa et al (10) performed an in vitro study to determine whether tensioning altered the biomechanical properties of grafts and reported that excessive graft tensioning decreased tensile resistance of tendons, although it reduced the elongation of the graft (10). The current study indicated that the strength of $\gamma$-irradiated deep frozen tendon grafts decreased when the pretension level reached $320 \mathrm{~N}$, implying that the tensile strength of the grafts may not improve with the increase of the pretension level. When the pretension level was set at 80 or $160 \mathrm{~N}$, the tensile strength of tendon allografts was closer to the native ACL at 1730-2160 N, which has been reported previously $(27,28)$.

The present study has certain limitations. Firstly, only tendon allografts with a diameter of $7 \pm 0.5 \mathrm{~mm}$ were included. Whether the outcomes for grafts with different diameters are consistent with the results obtained in the presents study remains to be elucidated. Secondly, only isotonic load was applied during the pretension process in the present study. Whether cyclic load protocols produce equivalent results requires further investigation. Thirdly, in vivo studies were not carried out and whether the clinical outcomes of ACL reconstruction could be improved based on the present study remains to be verified.

In conclusion, the results suggested that increasing the initial tension could effectively reduce the loss of stiffness due to viscoelasticity for the $\gamma$-irradiated deep frozen allogeneic tendon grafts. However, overloaded initial tension may decrease the tensile strength.

\section{Acknowledgements}

Not applicable.

\section{Funding}

No funding was received.

\section{Availability of data and materials}

The datasets used and/or analyzed during the current study are available from the corresponding author on reasonable request.

\section{Authors' contributions}

$\mathrm{XZ}$ and SG made substantial contributions to conception and design. WX and $\mathrm{XZ}$ performed the experiments and were the major contributors in writing the manuscript. JG and $\mathrm{YH}$ obtained the patient data regarding the diffuse brain injury. MC and YF made substantial contributions to analysis and interpretation of data. All authors read and approved the final manuscript.

\section{Ethics approval and consent to participate}

Not applicable.

\section{Patient consent for publication}

Not applicable.

\section{Competing interests}

The authors declare that they have no competing interests.

\section{References}

1. Jiang C, Chen G, Chen P, Li W, Zhang $H$ and Zhang W: Double-bundle revision anterior cruciate ligament reconstruction is effective in rescuing failed primary reconstruction and re-introducing patients to physical exercise. Exp Ther Med 15: 2074-2080, 2018.

2. Chia ZY, Chee JN, Bin-Abd-Razak HR, Lie DT and Chang PC: A comparative study of anterior cruciate ligament reconstruction with double, single, or selective bundle techniques. J Orthop Surg (Hong Kong) 26: 2309499018773124, 2018.

3. Jaglowski JR, Williams BT, Turnbull TL, LaPrade RF and Wijdicks CA: High-load preconditioning of soft tissue grafts: An in vitro biomechanical bovine tendon model. Knee Surg Sports Traumatol Arthrosc 24: 895-902, 2016.

4. Blythe A, Tasker T and Zioupos P: ACL graft constructs: In-vitro fatigue testing highlights the occurrence of irrecoverable lengthening and the need for adequate (pre)conditioning to avert the recurrence of knee instability. Technol Health Care 14: 335-347, 2006.

5. Elias JJ, Kilambi S and Ciccone WJ II: Tension level during preconditioning influences hamstring tendon graft properties. Am J Sports Med 37: 334-348, 2009.

6. Elias JJ, Rai SP and Ciccone WJ II: In vitro comparison of tension and stiffness between hamstring tendon and patella tendon grafts. J Orthop Res 26: 1506-1511, 2008.

7. Nurmi JT, Kannus P, Sievänen H, Järvelä T, Järvinen M and Järvinen TL: Interference screw fixation of soft tissue grafts in anterior cruciate ligament reconstruction: part 2: Effect of preconditioning on graft tension during and after screw insertion. Am J Sports Med 32: 418-424, 2004. 
8. Arneja S, McConkey MO, Mulpuri K, Chin P, Gilbart MK, Regan WD and Leith JM: Graft tensioning in anterior cruciate ligament reconstruction: A systematic review of randomized controlled trials. Arthroscopy 25: 200-207, 2009.

9. Numazaki H, Tohyama H, Nakano H, Kikuchi S and Yasuda K: The effect of initial graft tension in anterior cruciate ligament reconstruction on the mechanical behaviors of the femur-graft-tibia complex during cyclic loading. Am J Sports Med 30: 800-805, 2002.

10. Figueroa D, Calvo R, Vaisman A, Meleán P and Figueroa F: Effect of tendon tensioning: An in vitro study in porcine extensor tendons. Knee 17: 245-258, 2010.

11. Guillard C, Lintz F, Odri GA, Vogeli D, Colin F, Collon S, Chappard D, Gouin F and Robert H: Effects of graft pretensioning in anterior cruciate ligament reconstruction. Knee Surg Sports Traumatol Arthrosc 20: 2208-2213, 2012

12. Yoshiya S, Andrish JT, Manley MT and Bauer TW: Graft tension in anterior cruciate ligament reconstruction. An in vivo study in dogs. Am J Sports Med 15: 464-470, 1987.

13. Peña E, Martínez MA, Calvo B, Palanca D and Doblaré M A finite element simulation of the effect of graft stiffness and graft tensioning in ACL reconstruction. Clin Biomech (Bristol, Avon) 20: 636-644, 2005

14. Guo L, Chen H, Luo JM, Yang L, Gu LC and Fu DJ: An arthroscopic second-look study on the effect of remnant preservation on synovialization of bone-patellar tendon-bone allograft in anterior cruciate ligament reconstruction. Arthroscopy 32 868-877, 2016.

15. Carter TR and Rabago MT: Allograft anterior cruciate ligament reconstruction in patients younger than 25 years. J Knee Surg 29: 322-328. 2016.

16. Tian S, Wang B, Liu L, Wang Y, Ha C, Li Q, Yang X and Sun K: Irradiated hamstring tendon allograft versus autograft for anatomic double-bundle anterior cruciate ligament reconstruction: midterm clinical outcomes. Am J Sports Med 44 $2579-2588,2016$

17. Curran AR, Adams DJ, Gill JL, Steiner ME and Scheller AD: The biomechanical effects of low-dose irradiation on bone-patellar tendon-bone allografts. Am J Sports Med 32: 1131-1135, 2004.

18. Vyas D, Rabuck SJ and Harner CD: Allograft anterior cruciate ligament reconstruction: Indications, techniques, and outcomes. J Orthop Sports Phys The 42: 196-207, 2012.

19. Marrale J, Morrissey MC and Haddad FS: A literature review of autograft and allograft anterior cruciate ligament reconstruction. Knee Surg Sports Traumatol Arthrosc 15: 690-704, 2007

20. Shaerf DA, Pastides PS, Sarraf KM and Willis-Owen CA Anterior cruciate ligament reconstruction best practice: A review of graft choice. World J Orthop 5: 23-29, 2014.
21. Zheng X, Li T, Wang J, Dong J and Gao S: Medial collateral ligament reconstruction using bone-patellar tendon-bone allograft for chronic medial knee instability combined with multi-ligament injuries: A new technique. J Orthop Surg Res 11: 85, 2016.

22. Schwartz HE, Matava MJ, Proch FS, Butler CA, Ratcliffe A, Levy M and Butler DL: The effect of gamma irradiation on anterior cruciate ligament allograft biomechanical and biochemical properties in the caprine model at time zero and at 6 months after surgery. Am J Sports Med 34: 1747-1755, 2006.

23. Fideler BM, Vangsness CT Jr, Lu B, Orlando C and Moore T: Gamma irradiation: Effects on biomechanical properties of human bone-patellar tendon-bone allografts. Am J Sports Med 23: 643-646, 1995.

24. Ren D, Sun K, Tian S, Yang X, Zhang C, Wang W, Huang H, Zhang J and Deng Y: Effects of gamma irradiation and repetitive freeze-thaw cycles on the biomechanical properties of human flexor digitorum superficialis tendons. J Biomech 45: 252-256, 2012.

25. DiBartola AC, Everhart JS, Kaeding CC, Magnussen RA and Flanigan DC: Maximum load to failure of high dose versus low dose gamma irradiation of anterior cruciate ligament allografts: A meta-analysis. Knee 23: 755-762, 2016.

26. Suggs J, Wang C and Li G: The effect of graft stiffness on knee joint biomechanics after ACL reconstruction-a 3D computational simulation. Clin Biomech (Bristol, Avon) 18: 35-43, 2003.

27. Woo SL, Hollis JM, Adams DJ, Lyon RM and Takai S: Tensile properties of the human femur-anterior cruciate ligament-tibia complex. The effects of specimen age and orientation. Am J Sports Med 19: 217-225, 1991.

28. Noyes FR and Grood ES: The strength of the anterior cruciate ligament in humans and Rhesus monkeys. J Bone Joint Surg Am 58: 1074-1082, 1976.

29. Jackson DW, Corsetti J and Simon TM: Biologic incorporation of allograft anterior cruciate ligament replacements. Clin Orthop Relat Res 324: 126-133, 1996.

30. Gorschewsky O, Browa A, Vogel U and Stauffer E: Clinico-histologic comparison of allogenic and autologous bone-tendon-bone using one-third of the patellar tendon in reconstruction of the anterior cruciate ligament. Unfallchirurg 105: 703-714, 2002 (In German).

31. Mikami S, Yasuda K, Katsuragi R, Aoki Y, Minami A and Tohyama H: Reduction of initial tension in the in situ frozen anterior cruciate ligament. Clin Orthop Relat Res 419: 207-213, 2004.

This work is licensed under a Creative Commons Attribution-NonCommercial-NoDerivatives 4.0 International (CC BY-NC-ND 4.0) License. 